Article

\title{
Profiling of Signaling Pathways in Human Dermal Blood and Lymphatic Endothelial Cells Induced by Snake Venom Cyste- ine-Rich Secretory Protein (svCRiSP) from North American Snakes
}

\author{
Montamas Suntravat ${ }^{1,2, *}$, Oscar Sanchez ${ }^{1}$, Armando Reyes ${ }^{1}$, Abcde Cirilo ${ }^{1}$, Jack S. Ocheltree ${ }^{1}$, \\ Jacob A. Galan ${ }^{1,2}$, Emelyn Salazar ${ }^{1}$, Peter Davies ${ }^{3}$ and Elda E. Sanchez ${ }^{1,2}$
}

Citation: Suntrava, M. Sanchez, O Reyes, A.; Cirilo, A.; Cirilo, J.S.; Galan, J.A.; Salazar, E.; Davies, P.;

Sanchez, E.E. Profiling of Signaling Pathways in Human Dermal Blood and Lymphatic Endothelial Cells Induced by Snake Venom CysteineRich Secretory Protein (svCRiSP) from North American Snakes. Proceedings 2021, 65, $\mathrm{x}$.

https://doi.org/10.3390/xxxxx

Received: 5 November 2020 Accepted: 16 December 2020

Published: 14 January 2021

Publisher's Note: MDPI stays neutral with regard to jurisdictional claims in published maps and institutional affiliations.

Copyright: (c) 2021 by the authors. Submitted for possible open access publication under the terms and conditions of the Creative Commons Attribution (CC BY) license (http://creativecommons.org/licenses /by/4.0/).

1 National Natural Toxins Research Center (NNTRC), Texas A\&M University-Kingsville, MSC 224, 975 West Avenue B, Kingsville, TX 78363, USA; montamas.suntravat@etamuk.edu (M.S.); Oscar.Sanchez@tamuk.edu (O.S.); armando.reyes@students.tamuk.edu (A.R.); abcde.cirilo@students.tamuk.edu (A.C.); jack.ocheltree@students.tamuk.edu (J.S.O.); Emelyn.SalaJacob.Galan@tamuk.edu (J.A.G.); zarCastillo@tamuk.edu (E.S.); elda.Sanchez@tamuk.edu (E.E.S.)

2 Department of Chemistry, Texas A\&M University-Kingsville, MSC 161, Kingsville, TX 78363, USA

3 Institute of Biosciences and Technology, Texas A\&M University, Houston, TX, USA; pdavies@tamu.edu (P.D.)

* Correspondence: montamas.suntravat@tamuk.edu; Tel.: +1-361-593-3805

\begin{abstract}
Snake venom Cysteine-Rich Secretory Proteins (svCRiSPs) are important components in the venom of many snake species. Little is known about the contribution that they make to the local pathophysiology of snakebite. We investigated the role of svCRiSPs from the most medically significant species of North American snakes (Crotalus atrox, C. adamanteus, C. scutulatus scutulatus, C. horridus, and Agkistrodon piscivorus), focusing on the cellular and molecular mechanisms. We evaluated the biological activities of svCRiSPs (Catrox-CRiSP, Cada-CRiSP, Css-CRiSP, Chor-CRiSP, and App-CRiSP) by using both in vitro assays on human dermal lymphatic (HDLECs) and blood (HDBECs) endothelial cells permeability and in vivo Miles assay. Of all the CRiSPs tested, CssCRiSP and App-CRiSP displayed the highest increase in permeability compared to other crotaline CRiSPs. We initially screened the changes in protein expression and phosphorylation in HDLECs and HDBECs after treatment with Css-CRiSP and App-CRiSP using reverse phase protein arrays (RPPA). Studies are ongoing for identifying the key signaling that are involved in endothelial permeability after treatment with App-CRiSP and Css-CRiSP.
\end{abstract}

Keywords: signaling pathway; reverse phase protein arrays (RPPA); snake venom cysteine-rich secretory proteins (svCRiSPs); endothelial permeability; North American snakes

Key Contribution: Knowledge gained from these studies provides insights into the molecular mechanisms that underlie the effects of svCRiSPs on vascular function and contributes to a new level of understanding of the pathophysiology of snakebite

\section{Introduction}

Snake venoms are composed of complex mixtures of biological molecules. Among them, snake venom cysteine-rich secretory proteins (svCRiSPs) are ubiquitous components in the venom of many species of snakes [1,2], including Viperidae (Viperinae and Crotalinae) and Elapidae (Elapinae and Hydrophiinae) [3]. However, little is known of about the contribution that they make to the local pathophysiology of snakebite. Much of the information that has been garnered on the structure and biological activity of 
svCRiSPs has been derived from studies of Asian and Australian snakes (due in part to the concentration of snake venom research in these geographic areas). Much less is known of the biological activity of svCRiSPs present in the North American snakes' venom, especially rattlesnakes (Genus Crotalus) and cottonmouths (Genus Agkistrodon). Several of these svCRiSPs have been shown to block the activity of L-type $\mathrm{Ca}^{2+}$ and/or $\mathrm{K}^{+}$channels $[4,5]$. Two svCRiSPs, ES-CRiSP, isolated from an Asian pit viper, and natrin, isolated from cobra venom, have been shown to affect vascular endothelial cell activity [6,7]. Natrin from Naja atra has been shown to produce acute activation of MAP kinase signaling in human umbilical vein endothelial cells (HUVEC) [6]. ES-CRiSP from Echis carinatus sochureki, on the other hand, has not been reported to have an effect on MAP kinase signaling on its own but can block VEGF-dependent activation of Erk1/2 in HUVEC cells [7]. However, the mechanism of actions and molecular targets of most svCRiSPs remain unclear.

We have recently reported that Hellerin, a svCRiSP that we have isolated from the venom of the Southern Pacific Rattlesnake, C. oreganus helleri, increases vascular permeability in vivo and in vitro [8]. This study's objective was to investigate the biological activities of svCRiSPs isolated from the venoms of the most medically significant species of North American snakes. This study also aims to determine key singling pathways induced by svCRiSPs on human endothelial cells using reverse-phase protein microarray analysis (RPPA) analysis.

\section{Materials and Methods}

\subsection{Snake and Venom Collection}

The venoms were obtained from the National Natural Toxins Research Center (NNTRC), Texas A\&M University-Kingsville, Kingsville, TX, USA.

\subsection{Purification of CRiSPS}

svCRiSPs were isolated from crude venoms using two chromatographic steps consist of reverse phase C18 (Higgins Analytical PROTO 300 C18, $250 \times 4.6$ mm, $5 \mu \mathrm{m}$ column, Higgins Analytical, Inc., Mountain View, CA) and cation-exchange (Waters Protein-Pak ${ }^{\mathrm{TM}}$ SP 5PW, $7.5 \times 75 \mathrm{~mm}$ column, Waters Corp., Milford, MA) as previously described [8].

\subsection{SDS-PAGE Electrophoresis and N-Terminal Sequencing}

Purified CRiSPs were applied to NuPAGE® Novex 4-12\% (w/v) Bis-Tris SDS-PAGE gels (Invitrogen ${ }^{\mathrm{TM}}$, Carlsbad, CA, USA) using an XCell SureLock ${ }^{\mathrm{TM}}$ system $_{\text {(Invitrogen }}{ }^{\mathrm{TM}}$, Carlsbad, CA, USA). For the N-terminal sequencing, purified CRiSPs were transferred from an SDS-PAGE gel onto a PVDF membrane and stained with Coomassie brilliant blue $\mathrm{R}$-250. The sample membrane was processed for $\mathrm{N}$-terminal amino acid sequencing using Edman degradation method on a PPSQ-33B protein sequencer (SHIMADZU, Kyoto, Japan) following the manufacturer's instructions.

\subsection{LC-MS/MS Analysis and Proteomics Data Processing}

Venom protein identification and sequence analysis of CRiSPs (Css-CRiSP from C. s. scutulatus, Catrox-CRiSP from C. atrox, Cada-CRiSP from C. adamanteus, Chor-CRiSP from C. horridus, and App-CRiSP from A. p. piscivorus) were performed by LC-MS/MS mass spectrometry following the protocol as described by Suntravat et al. [8]. N-terminal amino acid sequences and sequences generated by MS were compared to the sequences in the GenBank database using BLASTX programs. Multiple alignments of the complete amino acid sequences were performed with a ClustalW program.

\subsection{Effect of CRiSPs on Vascular Permeability and Endothelial Cell Function}

Assays were performed as described previously [8]. Values are presented as fold increase with respect to negative control. 


\subsection{Reverse Phase Protein Array Analysis}

RPPA analyses were performed at the Functional Proteomic RPPA Core Facility at MD Anderson Cancer Center (Houston, USA) as described at https://www.mdanderson.org/research/research-resources/core-facilities/functional-proteomics-rppa-core/education-and-references.html. Heatmaps were generated using XLSTAT software version 2020.4.1. Proteins were ordered by the rank sum of the normalized values.

\section{Results and Discussion}

\subsection{Proteomic Analysis and Purification of CRiSPs}

Crotaline venoms are a rich and untapped resource for the discovery of new svCRiSPs that are useful in probing the cellular and molecular mechanisms involved in the effects of this family of toxins and on both lymphatic and blood vascular endothelial cell biology. To evaluate the venom composition of the C. atrox, C. adamanteus, C. s. scutulatus, C. horridus, and A. p. piscivorus used in this study, we analyzed the proteome of the venom using LC-MS/MS analysis. CRiSPs were identified to be $1.9-4 \%$ of the total venom proteomes (Figure 1, panels a-e). This observation corresponded to the previous proteomic and transcriptomic studies of these snakes [9-13].

We have successfully purified svCRiSPs from these crotaline venomous snakes using a two-step chromatographic protocol consisting of a reverse phase C18 HPLC chromatography, followed by SP-5PW cation exchange chromatography (Figure 1, a-e). Purified CRiSPs showed molecular mass of approximately $25 \mathrm{kDa}$ under non-reducing conditions (Figure 1f) and were verified as CRiSPs by N-terminal automated Edman Degradation amino acid sequencing.

a)

b)

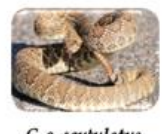

C. s. scutulatus

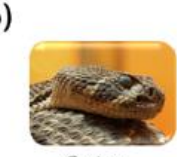

c)
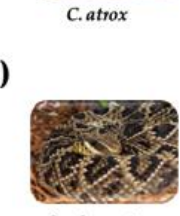

d)

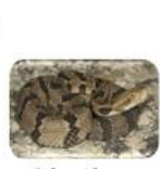

$$
\text { C. horridus }
$$

e)

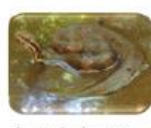

A. p. piscivorus
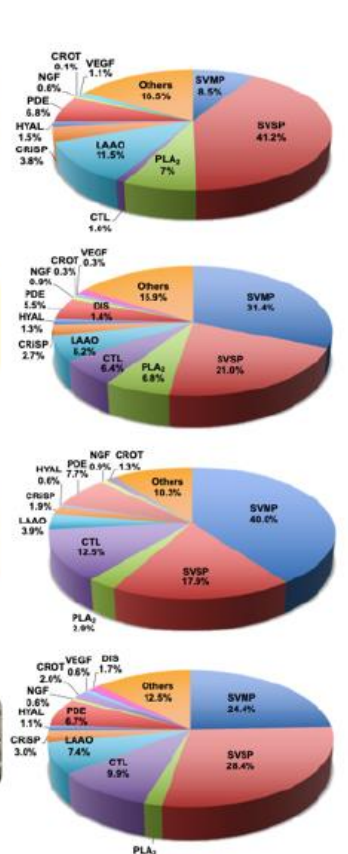

紫

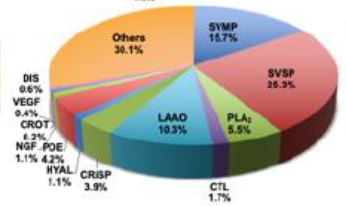

Reverse phase C18
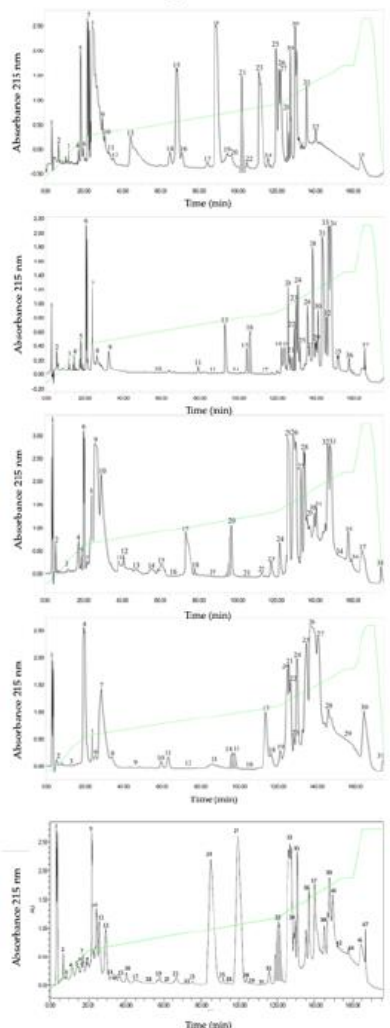
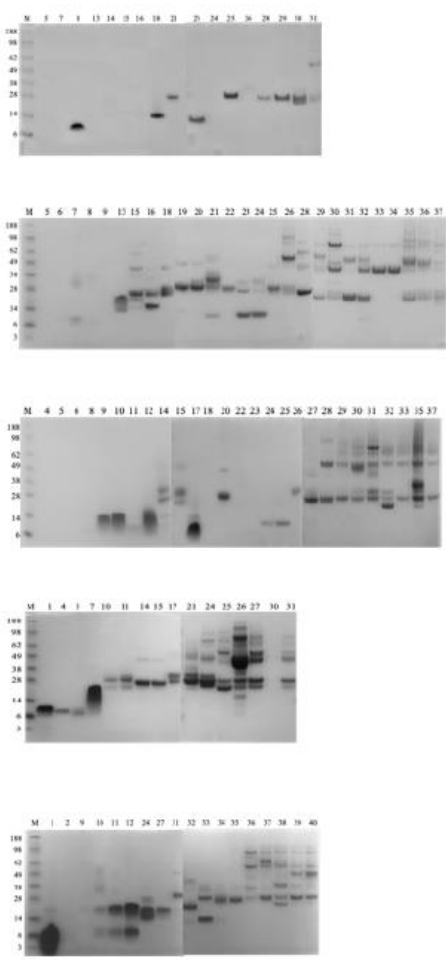

SP cation-exchange
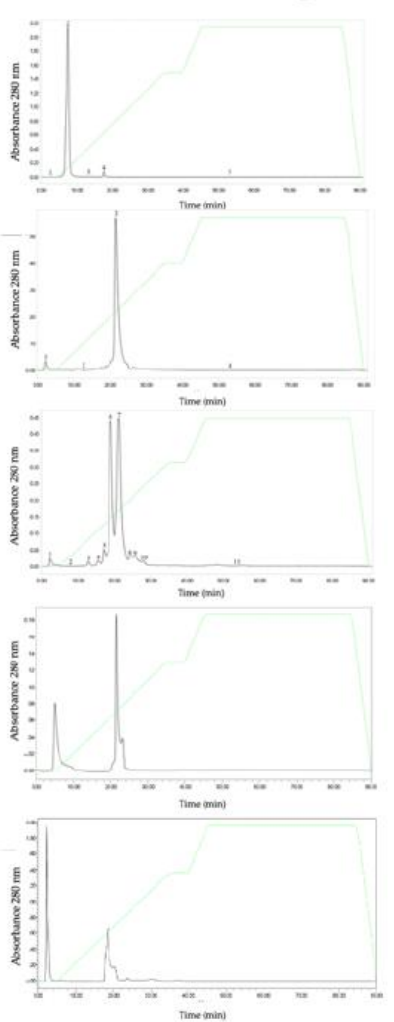
f)

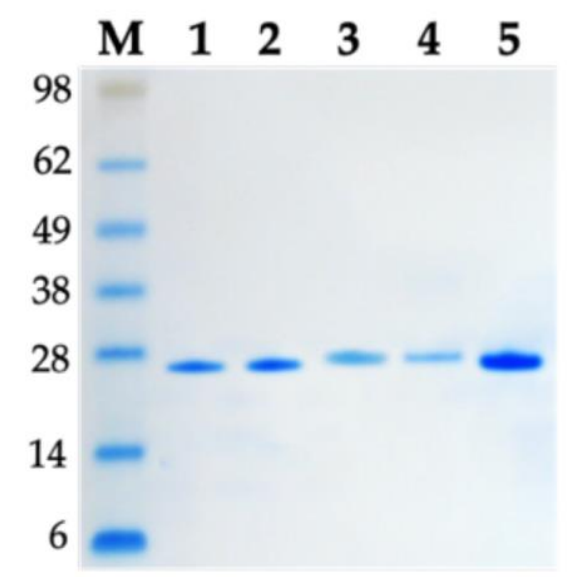

Figure 1. Venomics and purification steps of CRiSPs from five North American snakes (Panels a-e). Relative abundance of different protein families in each venom were identified by LC-MS/MS and are shown in pie charts. (f) SDS-PAGE of purified CRiSPs. M: SeeBlue Plus2 Markers (Invitrogen ${ }^{\mathrm{TM}}$ ). Lane 1: App-CRiSP; lane 2: Catrox-CRiSP; lane 3: Css-CRiSP; lane 4: Chor-CRiSP; lane 5: Cada-CRiSP.

\subsection{Amino Acid Sequences of CRiSPs}

Our CRiSPs shared a high similarity (80\%-97\%) to each other, and $70 \%-72 \%$ to elapid CRiSPs (Figure 2). Subtle variations in the conformations and amino acid residues could be responsible for their distinct biological functions.

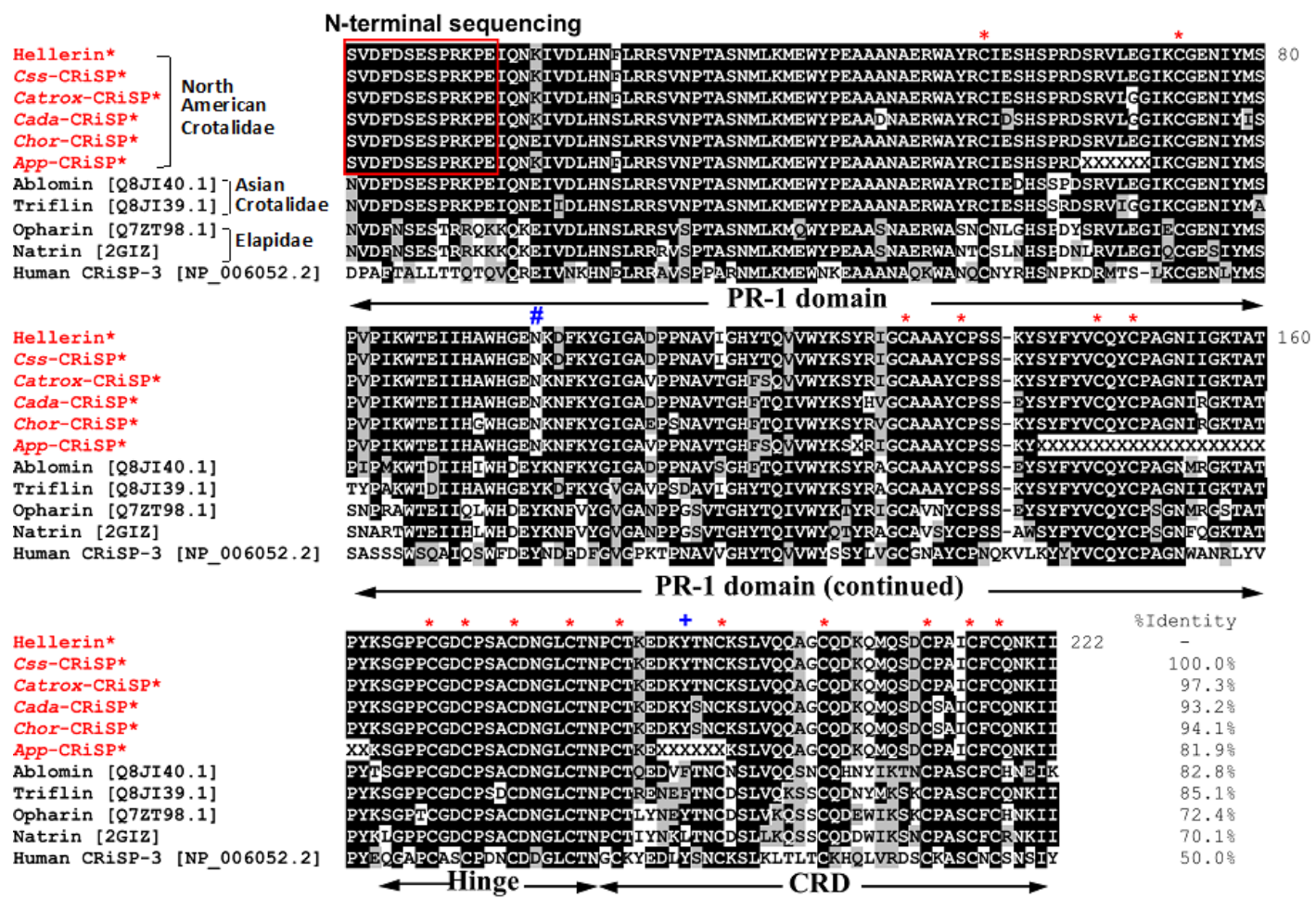

Figure 2. Multiple alignments of the amino acid sequence of svCRiSPs with other homologous venom proteins. All cysteine residues (an asterisk above the sequences) are conserved.

\subsection{Effect of CRiSPs on Vascular Permeability and Endothelial Cell Function}

In vivo Miles assay, subcutaneous injection of Css-CRiSP and App-CRiSP produced a significant rapid increase $(59 \%)$ in the trans-capillary leakage of an intravascular dye 
(Evans blue) compared to the saline control (Figure 3). App-CRiSP and Chor-CRiSP increased the vascular permeability to $33 \%$ and $26 \%$, respectively. Interestingly, neither Catrox-CRiSP nor Cada-CRiSP was active in the Miles assay.

\section{Vascular permeability induced by svCRiSPs using Miles assay}

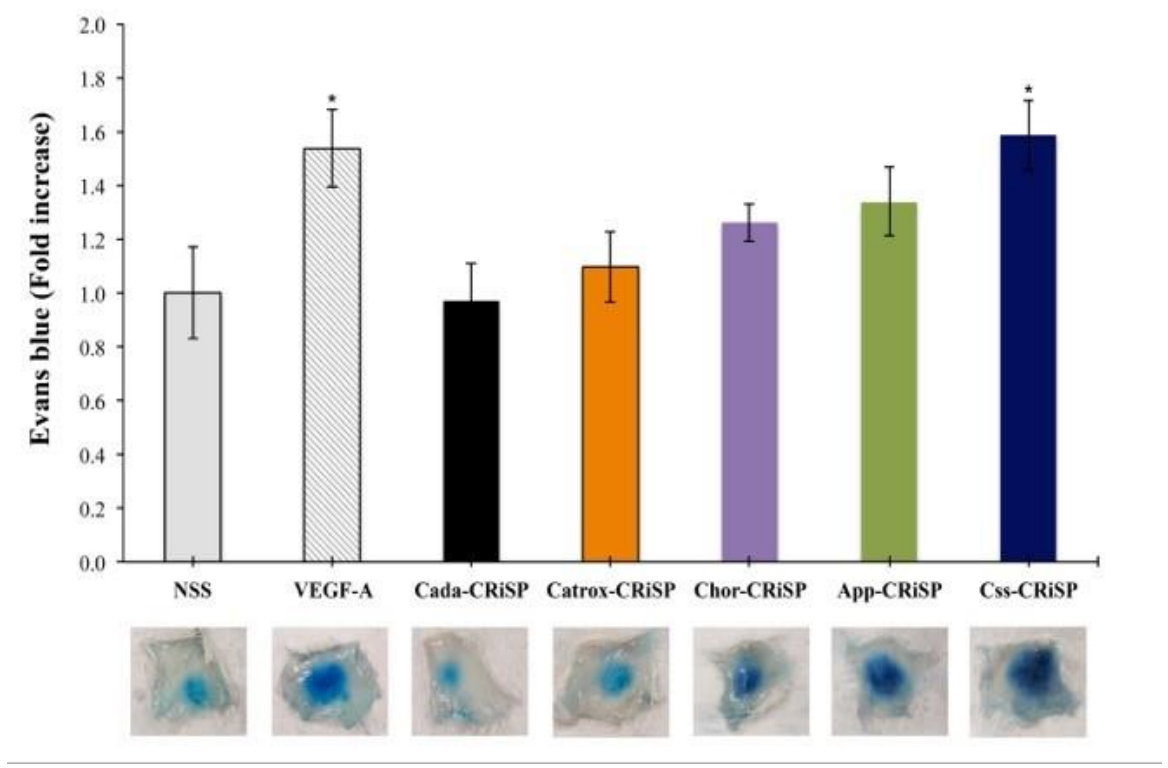

Figure 3. Quantification of an in vivo permeability assay was performed using the modified Miles assay. The results are presented as the fold change in OD for the agent versus vehicle (error bars: $\mathrm{SE})$.

To further analyze the effect of CRiSP on blood and lymphatic cell permeability, we used HDBECs and HDLECs in a cell permeability assay (Figure 4). App-CRiSP, CssCRiSP, and Catrox-CRiSP caused a significant and dose-dependent increase in HDBEC permeability at $1.3 \mu \mathrm{M}$ after $1 \mathrm{~h}$ of administration (Figure 4a). At $1.3 \mu \mathrm{M}$, Css-CRiSP, Catrox-CRiSP, and App-CRiSP but not Cada-CRiSP and Chor-CRiSP, significantly induced trans-endothelial permeability in HDLECs in comparison with that of cells treated with the PBS control (Figure 4b). Of all the CRiSPs tested, Css-CRiSP and App-CRiSP displayed the highest increase in acute vascular and endothelial permeability compared to other crotaline CRiSPs.

a) HDBECs

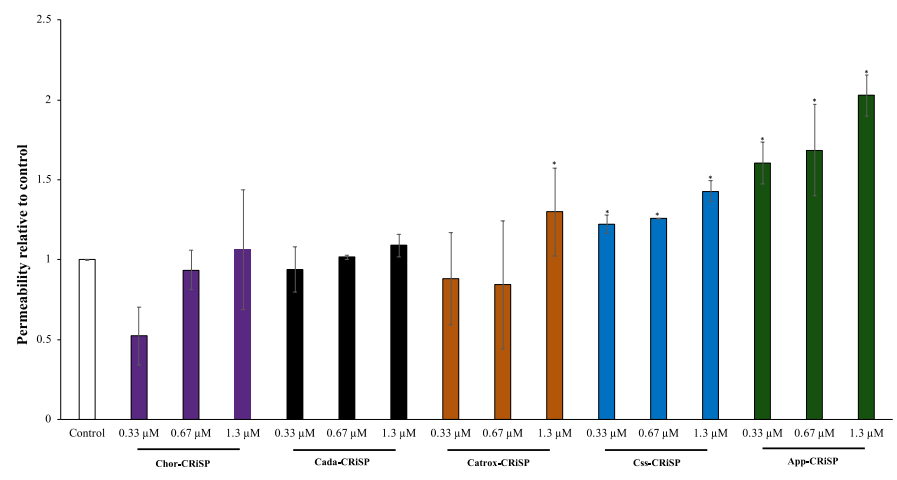

b) HDLECS

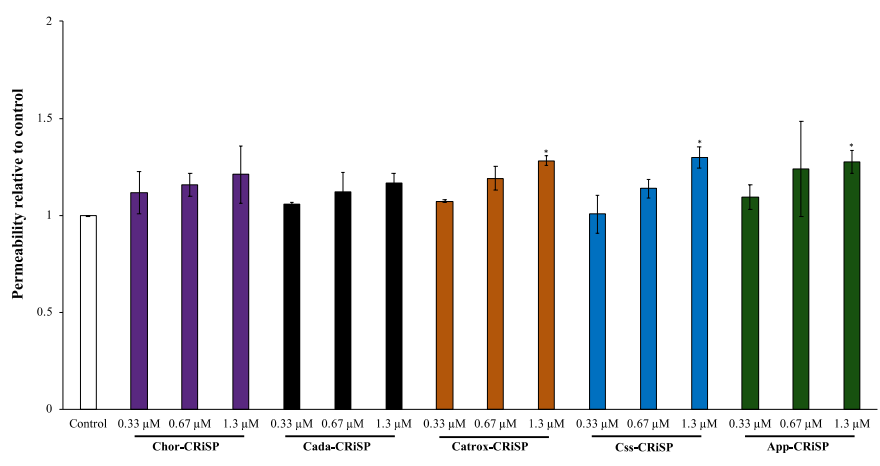

Figure 4. Effect of CRiSPs on monolayer barrier function of HDBECs (a) and HDLECs (b). Data expressed as mean \pm SD of two individuals experiments $(n=3) .{ }^{*} p<0.05$, compared with untreated control. 
3.4. Analysis of CRiSPs Effect on Protein Expression Patterns in HDBECs and HDLECs by RPPA Analysis

We used RPPA to screen and identify the key signaling that are involved in endothelial permeability after treatment with App-CRiSP and Css-CRiSP. Cell lysates were harvested after treated with PBS (control) or $1 \mu \mathrm{M}$ Css-CRiSP and App-CRiSP for $30 \mathrm{~min}$ and subjected to RPPA analysis of 439 proteins or protein phosphorylation using validated antibodies available in the Functional Proteomic RPPA Core Facility at MD Anderson Cancer Center. (Figure 5). Studies are ongoing for identifying the key signaling that are involved in endothelial permeability of HDLECs and HDBECs after treatment with AppCRiSP and Css-CRiSP.
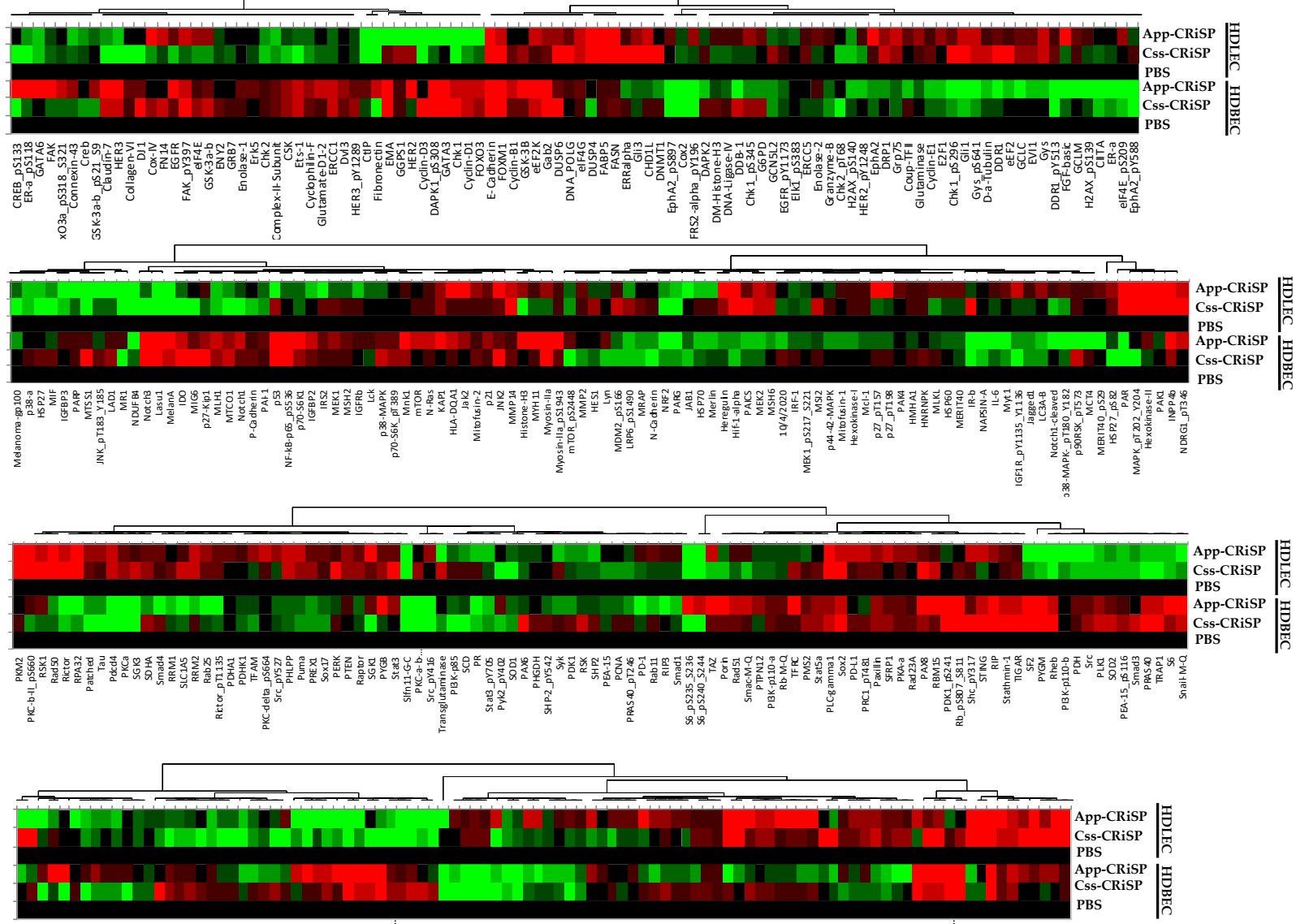

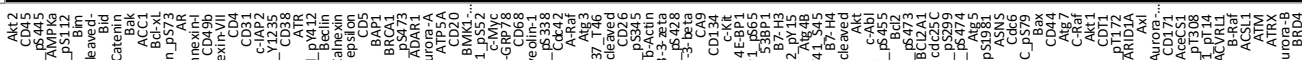
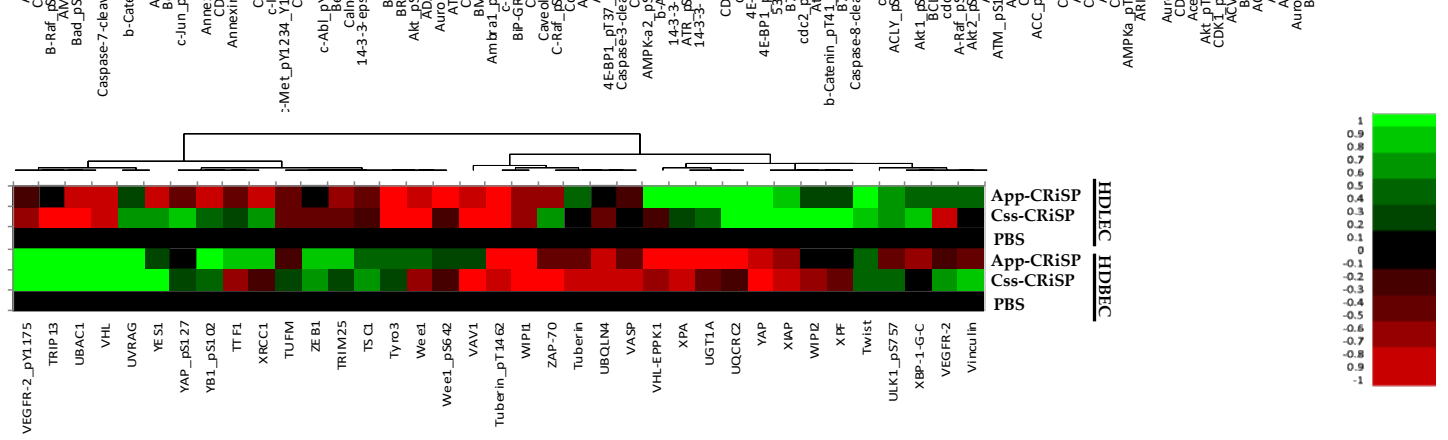

Figure 5. Heat map representation of RPPA analysis showing the changes in the protein expression in CRiSPs-treated HDBECs and HDLECs relative to the control group.

Using the RPPA platform offers a powerful approach to identify key signaling pathways involved in CRiSP dependent activation. However, the RPPA read out of a protein is mainly dependent on the antibody availability and quality. 


\section{Conclusions}

This study opens new avenues of exploration on the molecular targets of svCRiSPs. It should be relatively straightforward to extend the findings of these studies to other svCRiSPs and in the process gain additional insight into the mechanisms of action of the toxin and its role in the complex physiological mechanisms involved in snakebite envenomation.

Author Contributions: M.S., E.E.S., and P.D. designed the experiments. M.S., O.S., A.R., A.C., J.A.G., E.S.S., and E.S. conducted the experiments. M.S., A.R, J.S.O., and J.A.G. performed the bioinformatics analysis. M.S. analyzed the data and interpreted the data. M.S., J.A.G, P.D., and E.S.S. wrote and reviewed the manuscript.

Funding: Funding for the project was granted by the NIH/AREA, NIH/NHLBI grant\# 2R15HL137134-01 (Texas A\&M University-Kingsville, Dr. M. Suntravat), NIH/ORIP, Viper Resource Center grant\# 5P40OD010960-14 (NNTRC, Texas A\&M University-Kingsville, Dr. E.E. Sánchez), the Texas A\&M University's Presidential Undergraduate Research Program, 2019-2020 and 2020-2021 Research Supports, College of Arts and Sciences (Texas A\&M University-Kingsville, Dr. M. Suntravat), and the Robert A. Welch Foundation Department, grant\# AC-0006 (TAMUKDepartment of Chemistry). M.D. Anderson Cancer Center Reverse Phase Protein Array (RPPA) Core facility is funded by NCI\# CA16672.

Acknowledgments: We would like to thank, Nora Diaz DeLeon, Mark Hockmuller (NNTRC Serpentarium curator) and all the NNTRC personnel. We thank Clifford Stephan from the Center for Translational Cancer Research for his support with RPPA submission process.

Conflicts of Interest: The authors declare no conflict of interest.

\section{References}

1. Gibbs, G.M.; O'Bryan, M.K. Cysteine rich secretory proteins in reproduction and venom. Soc Reprod Fertil Suppl. 2007, 65, 261267.

2. Sunagar, K.; Johnson, W.E.; O'Brien, S.J.; Vasconcelos, V.; Antunes, A. Evolution of CRISPs associated with toxicoferan-reptilian venom and mammalian reproduction. Mol Biol Evol. 2012, 29, 1807-1822.

3. Gutierrez, J.M.; Calvete, J.J.; Habib, A.G.; Harrison, R.A.; Williams, D.J.; Warrell, D.A. Snakebite envenoming. Nat Rev Dis Primers. 2017, 3, 17063. PubMed PMID: 28905944.

4. Suzuki, N.; Yamazaki, Y.; Brown, R.L.; Fujimoto, Z.; Morita, T.; Mizuno, H. Structures of pseudechetoxin and pseudecin, two snake-venom cysteine-rich secretory proteins that target cyclic nucleotide-gated ion channels: implications for movement of the C-terminal cysteine-rich domain. Acta Crystallogr D Biol Crystallogr. 2008, 64, 1034-1042.

5. Wang, F.; Li, H.; Liu, M.N.; Song, H.; Han, H.M.; Wang, Q.L.; Yin, C.C.; Zhou, Y.C.; Qi, Z., Shu, Y.Y.; Lin, Z.J.; Jiang, T. Structural and functional analysis of natrin, a venom protein that targets various ion channels. Biochem Biophys Res Commun. 2006, 351, 443-448.

6. Wang, Y.L.; Kuo, J.H.; Lee, S.C.; Liu, J.S.; Hsieh, Y.C.; Shih, Y.T.; Chen, C.J.; Chiu, J.J.; Wu, W.G. Cobra CRISP functions as an inflammatory modulator via a novel $\mathrm{Zn} 2+-$ and heparan sulfate-dependent transcriptional regulation of endothelial cell adhesion molecules. J Biol Chem. 2010, 285, 37872-37883.

7. Lecht, S.; Chiaverelli, R.A.; Gerstenhaber, J.; Calvete, J.J.; Lazarovici, P.; Casewell, N.R.; Harrison, R.; Lelkes, P.I.; Marcinkiewicz, C. Anti-angiogenic activities of snake venom CRISP isolated from Echis carinatus sochureki. Biochim Biophys Acta. 2015, 1850 , 1169-1179.

8. Suntravat, M.; Cromer, W.E.; Marquez, J.; Galan, J.A.; Zawieja, D.C.; Davies, P.; Salazar, E.; Sánchez, E.E. The isolation and characterization of a new snake venom cysteine-rich secretory protein (svCRiSP) from the venom of the Southern Pacific rattlesnake and its effect on vascular permeability. Toxicon. 2019, 165, 22-30.

9. Lomonte, B.; Tsai, W.C.; Ureña-Diaz, J.M.; Sanz, L.; Mora-Obando, D.; Sánchez, E.E.; Fry, B.G.; Gutiérrez, J.M.; Gibbs, H.L.; Sovic, M.G.; Calvete, J.J. Venomics of New World pit vipers: genus-wide comparisons of venom proteomes across Agkistrodon. Journal of proteomics. 2014, 96, 103-116.

10. Calvete, J.J.; Fasoli, E.; Sanz, L.; Boschetti, E.; Righetti, P.G. Exploring the venom proteome of the western diamondback rattlesnake, Crotalus atrox, via snake venomics and combinatorial peptide ligand library approaches. Journal of proteome research. 2009, 8, 3055-3067.

11. Rokyta, D.R.; Lemmon, A.R.; Margres, M.J.; Aronow, K. The venom-gland transcriptome of the eastern diamondback rattlesnake (Crotalus adamanteus). BMC genomics. 2012, 13, 312. 
12. Rokyta, D.R.; Wray, K.P.; McGivern, J.J.; Margres, M.J. The transcriptomic and proteomic basis for the evolution of a novel venom phenotype within the Timber Rattlesnake (Crotalus horridus). Toxicon. 2015, 98, 34-48.

13. Matsunaga, Y.; Yamazaki, Y.; Hyodo, F.; Sugiyama, Y.; Nozaki, M.; Morita, T. Structural divergence of cysteine-rich secretory proteins in snake venoms. J Biochem. 2009, 145, 365-375. 